\title{
Entre exil et intégration, les populations migrantes parmi nous Une introduction
}

\author{
Jean-Bernard Racine, Lausanne
}

Migrations, processus d'intégration, relations interculturelles. Parcours migratoires, trajectoires, filières, mythes, territorialité perdue, territorialité retrouvée, histoires de vie. Quartiers ethniques, à nommer, villes et interculturalité, lieux de l'interculturalité dans l'espace urbain. Métropolisation, migrations internationales et pluralité des espaces sociaux, défis de l'intégration, contribution à la mobilité sociale et au capital humain. Opportunités internationales et migrations étudiantes, migrations et transferts de fonds. De beaux titres parfois, et quelle variété de problèmes, tous liés de près ou de loin au phénomène du mouvement de la population. Compte tenu de l'association, quasi consubstantielle de la présence de l'étranger à l'idée de ville, et plus encore aujourd'hui, à celle de la métropolisation, l'étude géographique des phénomènes migratoires est l'occasion peut-être de repenser, en même temps que la pensée migratoire, l'idée de ville, lieu de l'hétérogénéité. Une ville toujours rencontre et mosaïque, avant d'être institution, personnalité ou culture, voire spectacle et objet d'art, qui nous conduit à la question de savoir comment, à partir du moment où chacun cherche sa place dans la ville, aider l'étranger à «sortir» de sa sortie. Une hétérogénéité qui est justement l'apport à la société d'accueil, le plus souvent implicitement ou explicitement, attribué à l'immigration, la société d'accueil étant alors, comme l'a finement noté VÉRONIQUE DE RUDDER (1984), dotée d'homogénéité, d'unité, de cohérence et de solidarité interne. Versant positif de la dichotomie traditionnelle!

Chacun sait bien cependant que ce présupposé apport d'hétérogénéité à une société homogène, malheureusement à la base de différents modèles ou concepts proposés par les chercheurs en sciences sociales, ne correspond pas à la réalité des faits. Qu'une société d'accueil comme la Suisse n'est en aucun cas homogène, que c'est déjà une société complexe, formée de différents groupes et sous-groupes ayant tous leurs particularismes. Et que rien n'est plus dangereux que de se focaliser sur ce présupposé culturel en occultant les dimensions économiques, sociales, juridiques et politiques de nos différences, qui toutes jouent un rôle dans l'insertion d'un individu ou d'un groupe dans la société globale (DE Rudder \& Taboada-Leonetti 1982; RACINE 1998).

Une expérience, parmi d'autres, qui ont fait évoluer nos manières de concevoir l'ensemble des concepts dont nous avions besoin pour concevoir l'insertion de l'Autre dans le Même, et imaginer comment répondre à la question d'Alain Touraine (1997): Pourrons-nous vivre ensemble, égaux et différents? A l'évidence, l'assimilation ne se définit plus tout à fait de la même façon aujourd'hui qu'hier, non plus que l'intégration d'ailleurs, dont on reconnaît aujourd'hui, quand elle se borne à l'insertion socio-professionnelle ou aux structures de formation, qu'elle ne représente que son degré zéro (Ossipow 2000) sans que pour autant cette intégration soit réductible à la seule dimension socioéconomique puisqu'il est bien évident que les institutions comme le travail et l'école véhiculent les normes culturelles d'une société.

Ces concepts évoluent donc, se construisent, justement, se mesurent même parfois. Nous en avons besoin pour mettre de l'ordre dans nos construits. Mais songeons que le terme d'interculturalité n'a fait son apparition en Europe que depuis une vingtaine d'années, employé d'abord dans des contextes éducatifs et psychologiques. Aujourd'hui, on le découvre à tous les coins de rue, du domaine de la sociologie, de l'anthropologie des migrations, de la géographie humaine, et, singulièrement chez le signataire, de la géographie urbaine, que l'on étudie les pratiques, les représentations, ou les lieux mêmes. Avant même de définir les tenants et aboutissants de cette interculturalité, dont on aura compris qu'on en valorisait au départ le principe, à tort peut-être, nous avons fait l'hypothèse qu'il existait peut-être de ces lieux en villes, que dans un premier temps du moins nous avons imaginé en tant qu'interstices.

Comment concevoir, quel rôle peuvent ou pourraient jouer, dans la ville, ces lieux du «trans» ou du «co» comme aimait à les baptiser Alain Médam (1998), quelles questions poser et d'abord, plus simplement encore, au premier degré? Que savons-nous, quelle expérience pouvons-nous avoir de la possibilité de créer des liens sociaux à partir d'un lieu de rencontre entre personnes d'origine différentes, entre elles, et entre celles de la société d'accueil? Les questions peuvent se préciser beaucoup d'ailleurs, si on se demande dans quelle mesure une appartenance de genre ou/et des similitudes de vécus sont suffisantes pour rapprocher les migrants et les migrantes. En conséquence, que savons-nous des échanges réels entre participants? Peuvent-ils être considérés comme interculturels? D'abord, qu'est-ce que c'est que cette inter- 
culturalité? Quelque chose qui ressemble à notre interdisciplinarité ou à une éventuelle pluridisciplinarité, voire à une outre-disciplinarité, c'est-à-dire, audelà des barrières et des échanges, quelque chose de radicalement nouveau?

L'étranger dans la ville! Rapport à l'autre dans l'espace! Dans un monde de plus en plus marqué par l'hétérogénéité des populations urbaines, la place faite à l'étranger et l'aide à son intégration sont au cœur des problématiques de la ville d'aujourd'hui. Ville, donc espace urbain, tel qu'il est occupé, vécu, représenté, dans le registre des pratiques sociales comme dans celui des représentations symboliques. Espace partagé, interdit peut-être aussi, interdit à l'immigré, voire interdit par l'immigration quand il devient en quelque sorte un espace étranger à l'intérieur de la cité (TÉMINE 1997). Etrangers toujours porteurs d'un ailleurs qu'ils transportent avec eux et qui imposent dans nos villes, leur destination privilégiée depuis la nuit des temps, la réalité d'une différence fondée sur une altérité, certes plus ou moins grande, mais qui s'impose d'elle-même et qui conditionne en partie la vie de ceux qui sont différents (Simmel 1979). Comme en témoignent les pressions et manifestations politiques et sociales de tous ordres qui apparaissent de manière récurrente en cas d'afflux de nouveaux immigrants. Comme en témoignent heureusement aussi les efforts des uns et des autres pour penser, depuis une trentaine d'années maintenant, les structures d'accueil censées favoriser leur intégration ou leur assimilation, notions longtemps vagues et multivoques, mais qui furent progressivement construites, fut-ce à travers bien des «balbutiements» qui durent encore d'ailleurs. Sans que se rencontrent facilement pour autant les représentations des acteurs et des milieux concernés, que ces derniers soient immigrés ou institutionnels, "préposés» aux problèmes liés aux immigrés (dans le cadre des administrations fédérale, cantonales, municipales) à tous les niveaux de responsabilité, de décision ou de médiation.

Vieille histoire que ce lien privilégié entre ville et étrangers, étrangers et urbanité, que confirment toutes les statistiques et tous leurs corrélatifs. La question de la migration n'est pas propre à l'histoire contemporaine. On l'invoque pour expliquer que, dès l'apparition des préhominiens dans le Rift africain, il y a quelque trois millions d'années, les descendants de nos premiers ancêtres ont commencé progressivement à se répandre sur toutes les terres émergées. L'archéologie explique par elle les brusques modifications constatées dans l'outillage et les coutumes à certaines époques. Enfin, dès que nous possédons des archives écrites, la migration est présente. Et qui ne se souvient de l'instauration du Sabbat où nous entendons déjà évoquer ce même étranger qui lui non plus ne doit pas travailler ce jour-là, lui qui campe à l'intérieur des portes du campement des fils d'Israël? Mais l'exemple le plus remarquable est celui d'ABRAHAM, qui quitta le Nord de la Mésopotamie vers 1990 av. J.-C. pour un long périple, qui le mènera, lui, sa famille et ses troupeaux jusqu'en Egypte avant qu'il ne revienne mourir en Judée sans avoir pu s'établir, comme il le visait, dans le pays de Canaan. Il faudra à ses descendants un demi millénaire et un nouveau long séjour en Egypte pour y parvenir enfin. L'intégration est parfois problématique.

Dernier exemple enfin, la Grèce classique, celle de Platon, qui au Livre V des Lois (1951: 97) envisage l'émigration forcée au cas où la barque serait pleine à Athènes, précisément au cas où la population d'Athènes deviendrait supérieure à un «nombre d'or» fixé à cinq mille quarante foyers. Il précise d'ailleurs que

«si l'on arrive au comble de l'embarras pour ajuster les foyers au chiffre de cinq mille quarante et qu'il se produise un afflux excessif de citoyens par suite des sympathies nées de la cohabitation, dans cette extrémité on dispose de l'antique expédient que nous avons plusieurs fois mentionné, l'envoi de colonies, amis quittant des amis, en aussi grand nombre qu'il paraîtra nécessaire».

Dans le cas contraire, si la population arrive au-dessous du seul fixé, Platon envisage le recours à l'immigration. Donc rien de bien nouveau sous le soleil. L'idée de régulation systémique de la population était bien en germe depuis longtemps, reprise par MACHIAVEL pour qui le pouvoir se devait d'organiser les migrations pour répartir au mieux la population sur un territoire, ne pas le faire étant, selon lui, une erreur politique. Cette conception fut celle de l'Union soviétique et de tant de pays ayant tenté d'organiser leur politique d'aménagement du territoire, l'Indonésie par exemple. Elle l'est malheureusement encore pour des millions de gens dans pratiquement tous les pays du monde, qu'ils soient émetteurs ou récepteurs, ou qu'ils passent actuellement, comme jadis la Grèce, d'un état à l'autre.

Phénomène collectif, phénomène individuel, pour les pays et ceux qui en leur sein doivent accueillir et faire place à ceux qui cherchent une place, pour les pays qui laissent partir ou forcent à partir souvent les meilleurs de leurs enfants, le terme de migration recouvre tout à la fois un événement et un phénomène qui nous concerne tous à plusieurs degrés.

La Suisse, dont le tiers de la population est issue de l'immigration, n'échappe ni à ces processus, ni aux questions qu'ils posent à sa population, autochtone ou d'origine étrangère, en recherche d'une intégration véritable, dont le principe et la définition font encore largement débat, «tant sur le plan idéologique que 
scientifique» (Piguet 2004: 98). Renaissent en effet, autour de la question des requérants d'asile déboutés, de l'intégration de la Suisse dans l'Espace Schengen-Dublin, et de la liberté d'établissement, des discours offrant de bien désagréables réminiscences avec la période des votations xénophobes, voire de la période du tristement célèbre «la barque est pleine». Ce numéro spécial de Geographica Helvetica a pour but de nous aider à y voir plus clair, voire à confronter, dans le dialogue, nos représentations et nos perceptions, peut-être d'avancer sinon des solutions, du moins à jeter quelques ponts entre divers types de chercheurs.

L'ensemble des articles proposés dans ce numéro répond en grande partie à cet ensemble de questions. D'abord, et c'est toute logique, en offrant un tableau d'ensemble de la situation, double d'ailleurs. Le premier, qu'a bien voulu dresser WERNER HaUg sous le titre «Migrants and their descendants in Switzerland: an overview», le second, sous la plume d'ANTONIO DA Cunha, qui focalise son propos sur la porte d'entrée des migrations internationales montrant comment les grandes agglomérations suisses sont placées devant le défi de trouver des lignes d'action visant l'intégration de populations d'origine, de cultures et de religions de plus en plus diversifiées. Marina Marengo (2001) avait alors consacré sa thèse aux trajectoires migratoires en étudiant le cas des Italiens du Canton de Vaud avant de s'intéresser, en compagnie du soussigné aux "Lieux de l'interculturalité» (RACINE \& Marengo 1998; Racine 2000; Marengo 2000) au sein de la ville de Lausanne. Elle a l'occasion ici de développer sa pensée en nous livrant un certain nombre de notions attirant notre attention sur «la grande épaisseur de contenus cachés dans les parcours migratoires». A travers l'outil méthodologique des «histoires de vie» elle fait vivre cet entre-deux monde dont se nourrit sans doute le quotidien des migrants une fois installés en «terre promise», mais néanmoins sensibles au «mythe du retour». C'est justement cette dimension intra-urbaine des migrants installés qu'étudie, à la rencontre des concepts et méthodes de la sociologie et de la géographie, Isabelle CAPRANI. Autour du centre-ville niçois, celle-ci étudie de beaucoup plus près le lien tissé entre les étrangers et la ville, l'importante question de la nomination de quartiers centraux que l'on dira pourtant «ethniques» plutôt que «mixtes» et des significations qui lui seront liées, en prenant pour point de départ méthodologique cet énoncé-clé de JEAN Rémy (1990) voulant que la signification des lieux se saisisse à partir de leur usage. La dénomination «quartier transitoire» appliquée à ces quartiers apparaissant comme une cristallisation de tensions politique, économiques, sociologiques et culturelles, fera-t-elle fortune? L'avenir nous le dira.
En attendant, on découvrira avec intérêt deux articles consacrés à la dimension internationale des migrations. Le premier nous permet d'accueillir au sein de Geographica Helvetica, Allan Findlay qui signe l'article en compagnie d'Alexandra Stam et Enric RuIz-Gelices et il le fait en s'appuyant sur une importante enquête systématique en matière de mobilité étudiante conduite à l'origine par RuSsell King. C'est l'occasion d'illustrer, sur le plan qualitatif, l'importance du rôle des valeurs sociales et culturelles dans les migrations étudiantes, compte tenu aussi du poids des structures sociales conditionnant les réseaux de mobilité auxquels les étudiants peuvent avoir accès. En d'autres termes, une étude illustrant fort bien le point de vue d'Antony Giddens (1984: 25) quant aux relations entre structures et action humaine et la nécessité d'interpréter les propriétés des systèmes sociaux comme étant «both the medium and the outcome of the practices that constitue those systems».

Le second permet de s'interroger sur les retombées de ces migrations sur les pays «exportant» leur main d'œuvre et singulièrement, non plus seulement sur les «coûts», déjà fort analysés, mais sur les bénéfices les plus immédiats pour les pays sources d'immigration. Mathias Lerch et Philippe Wanner, du Forum suisse pour l'étude des migrations et de la population, nous ont offert à cet égard un fort étonnant tableau des caractéristiques démographiques, socio-économiques, migratoires et géographiques des ménages albanais bénéficiant de «remittances». Sans s'arrêter au seul niveau descriptif, ils complètent leur analyse à l'aide d'un modèle statistique mettant en évidence les facteurs intervenant sur la probabilité de recevoir des transferts de fonds. Ils démontrent en outre que la fonction première de ces transferts réside, avant tout, dans l'allégement de la pauvreté à l'échelle des ménages, mais que ces transferts peuvent également contribuer aux différentiels de développement économiques à l'échelle des régions.

\section{Bibliographie}

DE RUdDER, V. (1984): Trois situations de cohabitation pluri-ethnique à Paris. - In: Espaces et Sociétés 45: 43-59.

De Rudder, V. \& I. TABoAda-Leonetti (1982): La cohabitation pluri-ethnique: espace collectif, phénomènes minoritaires et relations sociales. - In: Pluriel 31: 37-54.

GidDens, A. (1984): The constitution of society: outline of the theory of structuration. - Cambrige: Polity Press.

MARENGo, M. (2000): Interculturality: a preferential path in the search for a new urban social equilibrium? - In: SCHNELL, I. (éd.): Urban segregation and desegregation. - Londres: Avebury: 87-103. 
MARENGo,M.(2001): Les trajectoires migratoires: entre flux, filières et mythes. Le cas des Italiens du canton de Vaud. - Travaux et recherches de l'Institut 21, Lausanne: Institut de géographie.

MÉdam, A. (1998): Villes pour un sociologue. - Paris: L'Harmattan.

Ossipow, L. (2000): Dire et penser la mixité: énoncés sur la «différence culturelle». - In: Centlivres, P. \& I. Girod (dir.): Les défis migratoires. Actes du colloque CLUSE «Les défis migratoires à l'aube du troisième millénaire», Neuchâtel, 1998. - Zurich: Editions Seismo: 472-480.

Piguet, E. (2004): L'immigration en Suisse. 50 ans d'entrouverture. - Collection Le savoir suisse 24, Lausanne: Presses polytechniques et universitaires romandes.

Platon (1951): Les Lois. - Paris: Les Belles Lettres.

Racine, J.-B. (1998): Proximités urbaines. Minorités ethniques et relations interculturelles. - In: Huriot, J.-M. (éd.): La ville ou la proximité organisée. - Paris: Anthropos: 85-85.

Racine, J.-B. (2000): Migrations, places and intercultural relations in cities revisiting our research. Critical reflections, methodological and normative lessons. In: SchNell, I. (éd.): Urban segregation and desegregation. - London: Avebury: 67-85.

Racine, J.-B. \& M. Marengo (1998): Migrations et relations interculturelles: les lieux de l'interculturalité. - In: Géographie et Cultures 25:22-37.
RÉmy, J. (1990): La ville cosmopolite et la coexistence inter-ethnique. - In: BASTENIER,A. \& F. Dassetto (éds): Immigrations et nouveaux pluralismes. Une confrontation de sociétés. - Bruxelles: De Boeck, Paris: Editions universitaires.

Simmel, G. (1979): Digressions sur l'étranger. - In: Grafmeyer, Y. \& I. Joseph: L'Ecole de Chicago. Naissance de l'écologie urbaine. - Paris: Aubier: 53-59.

TÉmine, E. (1997): Les immigrés dans la ville. - In: LAMIZET, B. \& P. SANSON (dir.): Les langages de la ville. - Marseille: Editions Parenthèses: 143-147.

Touraine, A. (1997): Pourrons-nous vivre ensemble, égaux et différents? - Paris: Fayard.

Prof. Dr. Jean-Bernard Racine, Institut de géographie, Université de Lausanne, Quartier Dorigny, Bâtiment Humense, $\mathrm{CH}-1015$ Lausanne.

e-mail: Jean-Bernard.Racine@unil.ch 